Research Paper

\title{
Detection of CTCs in portal vein was associated with intrahepatic metastases and prognosis in patients with advanced pancreatic cancer
}

\author{
Xiaoyu Liu ${ }^{1,2^{*}}$, Changyu $\mathrm{Li}^{1,2^{*}}$, Junhao $\mathrm{Li}^{1,2^{*}}$, Tianzhu $\mathrm{Yu}^{1,2}$, Guofeng Zhou ${ }^{1,3}$, Jiemin Cheng1,2, Guoping Li1,2, \\ Yang Zhou ${ }^{1,3}$, Wenhui Lou ${ }^{4,5}$, Xiaolin Wang ${ }^{1,2}$, Gaoquan Gong ${ }^{1,2}$, Lingxiao Liu ${ }^{1,2} \bowtie$,Yi Chen ${ }^{1,2 \bowtie}$ \\ 1. Shanghai Institute of Medical Imaging, Shanghai, 200032, China; \\ 2. Department of Interventional Radiology, Zhongshan Hospital Fudan University, Shanghai, 200032, China: \\ 3. Department of Radiology, Zhongshan Hospital Fudan University, Shanghai, 200032, China; \\ 4. Department of Pancreatic Surgery, Zhongshan Hospital Fudan University, 200032, China; \\ 5. Department of General Surgery, Zhongshan Hospital Fudan University, 200032, China. \\ *These authors contributed equally to this work. \\ $\square$ Corresponding authors: Yi Chen M.D. Ph.D, Department of Interventional Radiology, Zhongshan Hospital, Fudan University; Email: chen.yi@zs-hos \\ pital.sh.cn or Lingxiao Liu M.D. Ph.D, Department of Interventional Radiology, Zhongshan Hospital, Fudan University; Email: liu.lingxiao@zs-hospital.sh.cn or \\ Gaoquan Gong M.D. Ph.D, Department of Interventional Radiology, Zhongshan Hospital, Fudan University; Email: gong.gaoquan@zs-hospital.sh.cn \\ (c) Ivyspring International Publisher. This is an open access article distributed under the terms of the Creative Commons Attribution (CC BY-NC) license \\ (https:// creativecommons.org/licenses/by-nc/4.0/). See http://ivyspring.com/terms for full terms and conditions.
}

Received: 2017.11.23; Accepted: 2018.04.23; Published: 2018.05.22

\begin{abstract}
Pancreatic cancer is amongst the most lethal malignancies with increasing incidence and mortality worldwide. Distant metastases, especially intrahepatic metastases, is the leading cause of death for pancreatic cancer. Circulating tumor cells (CTCs) are neoplastic cells released from the primary tumor into circulation, and play critical roles in metastases of various types of cancers. Though clinical studies showed that detection of CTCs in peripheral circulation was associated with worse prognosis in patients with breast cancer and hepatocellular carcinoma, detection CTCs in peripheral blood of pancreatic cancer was still challenging due to hepatic filtration and technical limitations. Previous studies have demonstrated that CTCs could be detected in portal vein circulation in patients with pancreaticobiliary carcinoma. In the present study, taking advantage of ultrasonography-guided transhepatic puncture, we analysis CTCs in portal vein blood obtained from patients with advanced pancreatic cancer. CTCs were detected in all 29-portal vein blood of samples, and absolute numbers of circulating pancreatic cancer cells in portal vein was significantly higher than that in peripheral circulation. Furthermore, we found that CTC counts in portal vein was highly associated with intrahepatic metastases and indicated poorer prognosis in patients with advanced pancreatic cancer. Short-term expansion and in vitro drug sensitivity assay showed that CTCs derived from portal vein blood were highly resistant to several chemotherapy regimens. In summary, detection of CTCs in portal vein could be a powerful tool to stratify the risk of intrahepatic metastases of pancreatic cancer, and provided new insight into the biological feature of pancreatic cancer metastases and drug resistance.
\end{abstract}

Key words: pancreatic cancer, circulating tumor cells, portal vein, intrahepatic metastases

\section{Introduction}

Pancreatic cancer is one of the most lethal malignancies with increasing incidence and mortality worldwide[1]. Most patients with the disease are diagnosed at advanced stage, making them ineligible for curative treatment[2-4]. Even for patients with early stage disease, intrahepatic metastases were frequently occurred after curative resection[5-7]. In contrast, traditional chemotherapy and targeted therapy has been unsatisfactory in treatment of metastatic pancreatic cancer so far[8, 9]. Though 
clinical approaches, such as imaging, pathology and serum tumor markers were widely used to monitor disease progression currently, detection and assessment of early intrahepatic metastases are still challenging for pancreatic cancer[3]. There is an urgent need to discover more informative biomarkers to assess the tumor burden and intrahepatic metastatic risk of pancreatic cancer.

Circulating tumor cells (CTCs), shed from primary tumor into circulation, are considered precursors for metastases[10-12]. It has been explored as a less invasive biomarker for assessing solid tumor burden[13-15]. Previous studies have demonstrated that CTCs in peripheral circulation may serve as a predictive marker for metastases and overall survival in patients with breast cancer[16, 17], prostate cancer[13, 18-21], and hepatocellular carcinoma[22, 23]. Furthermore, Aceto et al revealed that CTCs were oligo-clonal precursors of breast cancer metastasis in vivo[16]. It is anticipated that quantification and molecular subtyping of CTCs could be adopted for monitoring tumor burden and metastasis of pancreatic cancer[24, 25]. However, CTCs are extremely rare with an estimates of one CTC per billion blood cells in patients with pancreatic cancer. Detection of rare CTCs in peripheral blood of pancreatic cancer was challenging with current available techniques [26, 27].Recently, Poruk et al analyzed CTCs of 60 resectable pancreatic cancer, only forty-six patients (77\%) were found CTCs positive with the median number of $4 \mathrm{CTC} / \mathrm{mL}$ in peripheral blood[28]. Importantly, bloodstream of pancreas flows through the portal vein into the liver, the hepatic filtration effect may reduce CTCs into peripheral circulation and increased the difficulty of detection CTCs in peripheral circulation[24, 27]. Catenacci et al first reported that portal CTCs are far more common in portal vein than in peripheral circulation in patients with pancreaticobiliary carcinoma[27], and they found portal vein blood contains more CTCs in pancreatic cancer patients. Though they did not further investigate the correlation between clinical features and portal vein CTCs because of the smaller sample size and heterogeneity of the patients, analysis of portal vein CTCs holds great promise for providing new insight into metastasis of pancreatic cancer.

In the present study, we analyzed CTCs in both portal vein and peripheral circulation in 29 patients with advanced pancreatic cancer. Our results showed that patients with metastatic disease has a significantly higher CTCs numbers in portal vein than patients with locally advanced disease. CTCs burden in portal vein was highly associated with prognosis of the patients. Furthermore, the results of the drug sensitivity test showed that CTCs from portal vein were highly resistant to several clinical regimens.

\section{Patients and Methods}

\section{Patients and study design}

29 patients with advanced pancreatic cancer were enrolled at Zhongshan Hospital Fudan University between February 2016 and January 2017. All patients received gemcitabine-based chemotherapy and arterial infusion chemotherapy at the medical center. A compete catalogue of clinical parameters, pathological data and radiographic data were collected from the electronic database of Zhongshan Hospital. Intrahepatic tumor burden was assessed according the radiography data. Tumor sites and maximum tumor size was assessed by two radiologist (GFZ and YZ). As described in our previous reports, follow-up was made by telephone call every 3 months by a full time clinical investigator. None of the patients lost to follow-up during the study. Vital status was obtained and survival time was estimated from the first treatment to death. Details of the chemotherapy or infusion chemotherapy treatment have been described previously. The study was approved by the Ethics Committee of Zhongshan Hospital Fudan University (No. B2014-098; No. B2017-048). The study was performed in accordance with the principles of Declaration of Helsinki.

\section{Samples Collection and Isolation of CTCs}

Fine-needle aspirates (FNA) biopsy was performed in standard procedure. Then we identified the intrahepatic portal vein under the ultrasonography guidance. A 20-guage needle was punctured into the portal vein trans-hepatically (Figure 1). 1 to 3 aliquots of $7.5 \mathrm{ml}$ blood samples were aspirated from portal-vein and draw into $10 \mathrm{ml}$ Streck preservation tubes (Streck 218962, USA). Peripheral blood was then collected in parallel and processed identically. The blood sample was processed using the ClearBridge ClearCell FX system ${ }^{\mathrm{TM}}$ (ClearBridge Biomedics, Singapore) [29]. Samples were further negatively sorted to remove the CD45 positive monocytes using EasySep ${ }^{\mathrm{TM}}$ Human CD45 Depletion Kit (18259RF, StemCell, USA). The identification of CTCs was based on immunofluorescence staining. CK19 or EpCAM positive cells was recognized as potential CTCs. Multicellular groupings of CTCs (CTC cellular $\geq 3$ ) was considered as CTC cluster.

\section{Drug sensitivity test of the CTCs}

After depletion of CD45 positive cells, CTCs was transferred into a $1.5 \mathrm{~mL}$ tube, and washed three times using $1 \times$ PBS (with $1 \%$ penicillin and 
streptomycin). Then the CTCs was transferred into ultra-low attachment 96-well plate for short-time expansion (2 4 days). Cell viability was assessed using the eBioscience ${ }^{\mathrm{TM}}$ Indo-1 AM Calcium Sensor Dye (65085639, Thermo Fisher) system. Cultured CTCs were incubated with 2 umol/L calcium dye system. With this dye and drug combination, viable CTCs are shown green and dead cells are dark. Imaging was performed with the NIKONE-C1 confocal microscope system.

\section{Statistical Analysis}

All data were entered using Epidata 3.1 software (Odense Denmark, EpiData Association ). Continuous data were presented as median or range, and compared using Wilcoxon test. Categorical data were presented as absolute and relative frequencies and compared using the chi-square test. Log-rank test was used to compare the survival between different patient groups. Two-sided $\mathrm{p}$ values less than 0.05 were considered statistically significant. The statistical analysis was conducted using SAS 9.2 (SAS Institute Inc, Cary, NC) and Graph Pad Prism 6.0 (Graph Pad Software Inc., La Jolla, CA, USA).

\section{Results}

\section{Patient characteristics}

$16(55 \%)$ of the 29 patients were locally advanced pancreatic cancer, and 13 (45\%) patients were with intrahepatic metastases disease. As described in the methods part, we collected portal vein blood from all 29 patients. In parallel, we collected 14 shares of paired peripheral blood sample.

None of the patients suffered bleeding after the procedure. $14(48 \%)$ subjects reported mild to moderate abdominal pain after the infusion chemotherapy. Our results showed that blood of portal vein could be safely collected by trans-hepatic puncture using 20G needle in clinic. The clinical characteristics of the 29 individuals were listed in Table 1.

Table 1. Clinical parameters of all included patients

\begin{tabular}{llllllll}
\hline Patient & Gen & Age & Intrahepatic & CA199c & CEAd & $\begin{array}{l}\text { CTC counts } \\
\text { (portal }\end{array}$ & $\begin{array}{l}\text { CTC counts } \\
\text { (peripheral) }\end{array}$ \\
ID & der $^{\mathrm{a}}$ & (year & Metastases & & & & \\
& & s) & & & & vein) & \\
\hline CTC1 & M & 67 & Y & 293 & 8.2 & 908 & 74 \\
CTC2 & M & 64 & N & 9.4 & 2.1 & 86 & 17 \\
CTC3 & F & 42 & Y & 14.2 & 2.4 & 8 & 0 \\
CTC4 & M & 76 & N & 39 & 0.9 & 174 & 1 \\
CTC5 & M & 45 & N & 134.2 & 3.3 & 43 & 1 \\
CTC6 & M & 57 & N & 16 & 2.6 & 114 & 35 \\
CTC7 & M & 61 & N & 188 & 7.5 & 704 & 0 \\
CTC8 & F & 58 & N & 28.4 & 151.3 & 131 & 26 \\
CTC9 & M & 71 & N & 10000 & 80.2 & 295 & 61 \\
CTC10 & F & 79 & N & 2 & 5.2 & 204 & 0 \\
CTC11 & M & 62 & N & 10000 & 9.3 & 41 & 0 \\
CTC12 & M & 50 & Y & 2159 & 3.4 & 822 & 62 \\
CTC13 & F & 74 & N & 601.1 & 2.7 & 107 & 0 \\
CTC14 & M & 66 & N & 2281 & NA & 106 & 17 \\
CTC15 & F & 55 & Y & 21.3 & 5.3 & 372 & N/A \\
CTC16 & M & 71 & Y & 135.5 & 1.8 & 305 & N/A \\
CTC17 & F & 62 & Y & 28.8 & 3 & 719 & N/A \\
CTC18 & F & 64 & Y & 446.9 & 6.6 & 394 & N/A \\
CTC19 & M & 62 & N & 1394 & 8.8 & 85 & N/A \\
CTC20 & F & 70 & N & 2760 & 4 & 47 & N/A \\
CTC21 & M & 57 & Y & 79.1 & 2.6 & 217 & N/A \\
CTC22 & M & 49 & Y & 10000 & 65.5 & 489 & N/A \\
CTC23 & F & 73 & Y & 2399 & 20.3 & 72 & N/A \\
CTC24 & M & 52 & N & 1200 & 29.2 & 215 & N/A \\
CTC25 & M & 72 & N & 29 & 5.6 & 104 & N/A \\
CTC26 & M & 69 & Y & 836.1 & 2.2 & 95 & N/A \\
CTC27 & F & 64 & N & 224 & 4.4 & 132 & N/A \\
CTC28 & M & 63 & Y & 346.9 & 3.3 & 336 & N/A \\
CTC29 & M & 69 & Y & 10000 & 18.6 & 845 & N/A \\
\hline
\end{tabular}

a:Male $=\mathrm{M} ; \mathrm{Female}=\mathrm{F}, \mathrm{b}: \mathrm{Yes}=\mathrm{Y} ; \mathrm{No}=\mathrm{N}$, c:pre-treatment $\mathrm{CA1} 199$ value $(\mathrm{U} / \mathrm{ml}), \mathrm{d}$ : pre-treatment CEA value $(\mathrm{ng} / \mathrm{ml})$, e: CTC counts per $7.5 \mathrm{ml}$ blood.
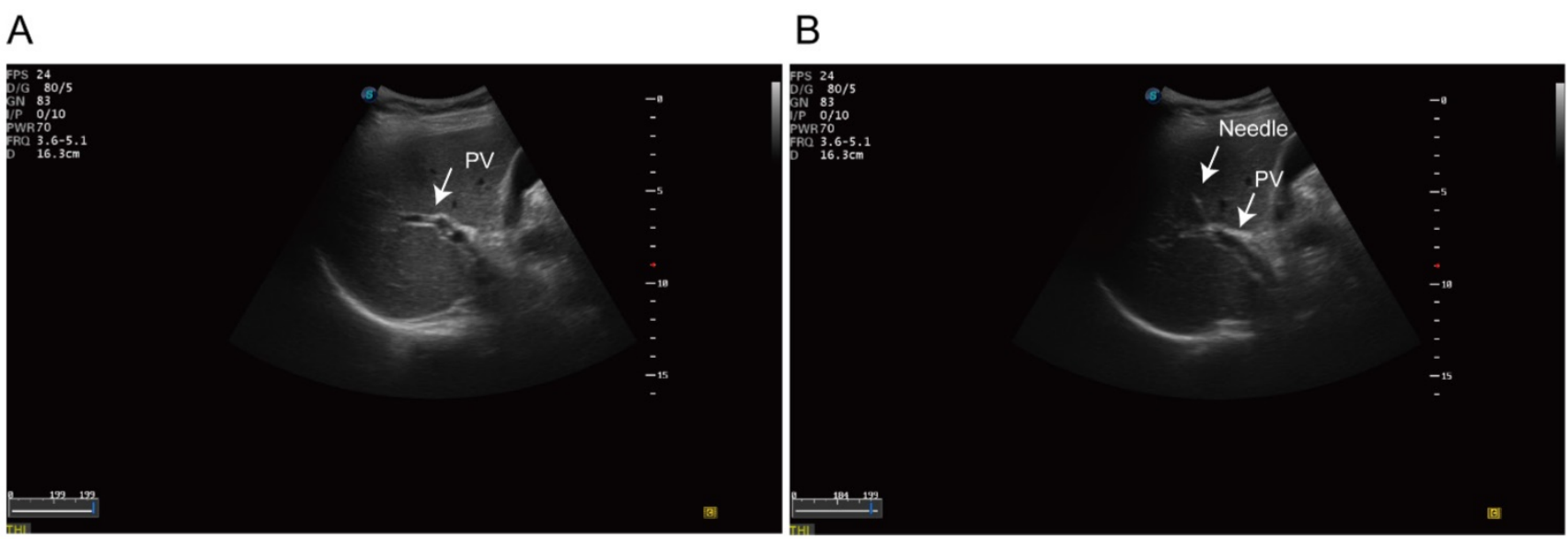

Figure 1. Ultrasonography-guided transhepatic puncture. (A) Identification of the portal vein under the ultrasonography guidance. (B) Transhepatic puncture of the portal vein using the 21 gauge needle. 


\section{CTCs counts in portal vein and peripheral circulation}

CTCs were detected in portal vein blood of all 29 patients, while only $8(8 / 14,54 \%)$ patients had detectable CTCs in peripheral circulation (Figure 2A,B). We also observed CTCs clusters in portal vein blood(Figure 2C), while no CTCs clusters were observed in peripheral blood samples. CTCs counts in portal vein was significantly higher than that in peripheral circulation $(282.0$ vs. 21.0 per $7.5 \mathrm{ml}$, $\mathrm{p}=0.0009$, Figure 2D). We further analyzed the correlation between clinical parameters and CTCs counts in portal vein. The average CTCs count in portal vein of metastatic patients was 449.0 per $7.5 \mathrm{ml}$, which was significantly higher than that in the 16 locally advanced patients (mean: 161.0 per $7.5 \mathrm{ml}$, $p=0.0054$, Figure 2B). We did not observed correlation between CTCs counts and other clinical parameters (Table 2, Table S1).CD133 is considered as a cancer stem cell marker for pancreatic cancer. We assessed the CD133 positive CTCs in portal and peripheral circulation, and the preliminary results showed that CD133 positive CTCs in portal vein ranges from $2.5 \% \sim 12 \%$, however no CD133 positive CTCs was observed in peripheral blood samples.

\section{CTCs counts in portal vein circulation was correlated with survival of the patients}

The median overall survival time for the 29 advanced patients was 13.5 (95\% CI: 11.8 17.6) weeks.
For 11 patients with portal vein CTCs less than 150 per $7.5 \mathrm{ml}$, median overall survival was 19.8 (95\% CI: 16.8 25.4) weeks, and for 17 patients with portal vein CTCs over 150 per $7.5 \mathrm{ml}$, median overall survival was 9.2 (95\% CI: 7.8 11.8) weeks. Overall survival was significant shorter in patients with portal vein CTCs over 150 per $7.5 \mathrm{ml}$ (log-rank $\mathrm{p}<0.0001$, Figure 3). We did not observe correlation between portal vein CTCs clusters and overall survival of the patients.

Table 2. Correlation between clinical parameters and CTCs counts in portal vein circulation.

\begin{tabular}{llc}
\hline Characteristics & CTC counts (mean,95\%CI) & $p$ value \\
\hline Gender & $315(150,398)$ & 0.3685 \\
Male & $219(174,456)$ & \\
Female & & \\
Age (years) & $268(70,466)$ & 0.8487 \\
$<60$ & $288(157,419)$ & \\
$>60$ & & 0.5789 \\
CA199 level (U/ml) & $217(25,408)$ & \\
$<37$ & $306(177,436)$ & 0.5039 \\
$>37$ & & \\
CEA level (pg/ml) & $220(89,354)$ & 0.3204 \\
$<5$ & $347(179,514)$ & \\
$>5$ & & $0.0004^{*}$ \\
Tumor Location & $340(182,498)$ & \\
Head and Neck & $210(75,344)$ & 0.9914 \\
Body and Tail & & \\
Intrahepatic Metastases & $449(368,530)$ & \\
Yes & $126(108,144)$ & \\
No & & \\
Portal Vein Invasion & $281(208,354)$ & \\
Yes & $282(216,348)$ & \\
No & &
\end{tabular}

A

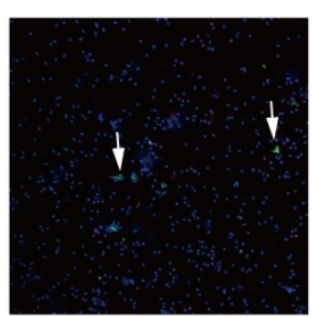

D

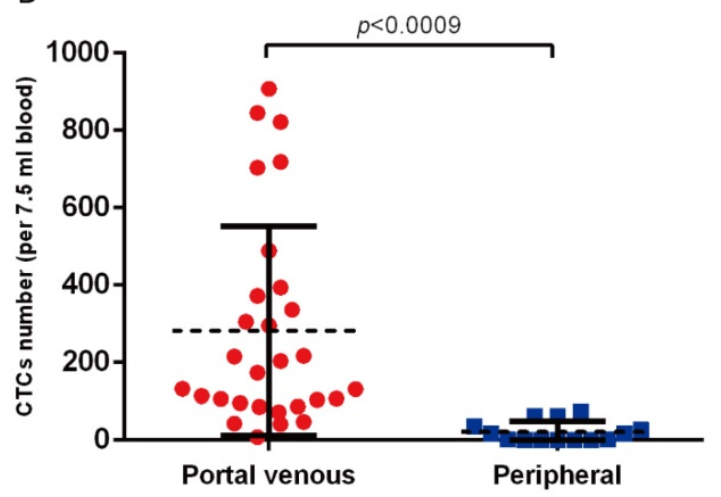

B

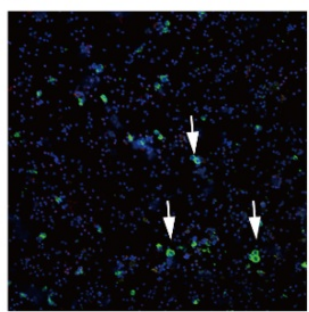

C

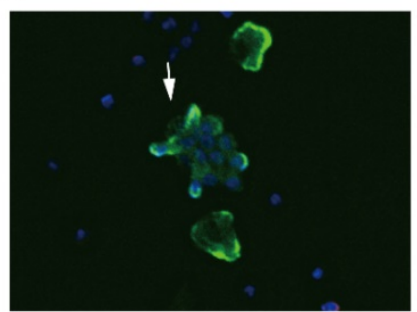

E

2. (A) Detection of CTCs in peripheral blood and (B) portal vein blood in patients with advanced pancreatic cancer. White arrows indicate the CK19 or EpCAM positive CTCs. (C) CTCs clusters derived from portal vein blood. (D) CTCs clusters are significantly higher in portal vein in patients with advanced pancreatic cancer. (E) Portal vein CTCs counts was significantly higher in patients with intrahepatic metastases. 


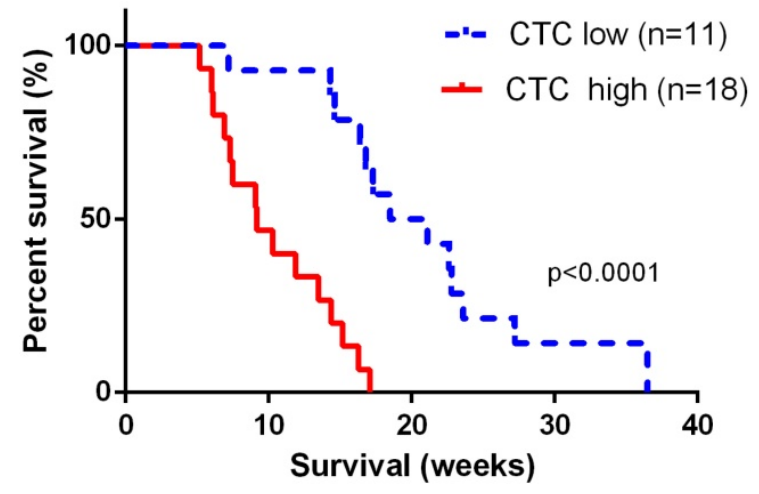

Figure 3. Overall survival was significant shorter in patients with portal vein CTCs over 150 per $7.5 \mathrm{ml}$. The CTC low groups represented patients with portal vein CTC counts $<150$ per $7.5 \mathrm{ml}$, and the CTC high group represented patients with CTC counts $\geq 150$ per $7.5 \mathrm{ml}$.

\section{Ex vivo culture and drug test system of CTC derived from portal vein}

Next, we tried to use CTCs to test drug sensitivity and a panel of single or drug combinations were included. The drug concentration was based on median inhibitory concentration levels identified in previous large-scale cancer cell line screens[30]. The drug test assay was succeeded in $7(7 / 29,24 \%)$

A

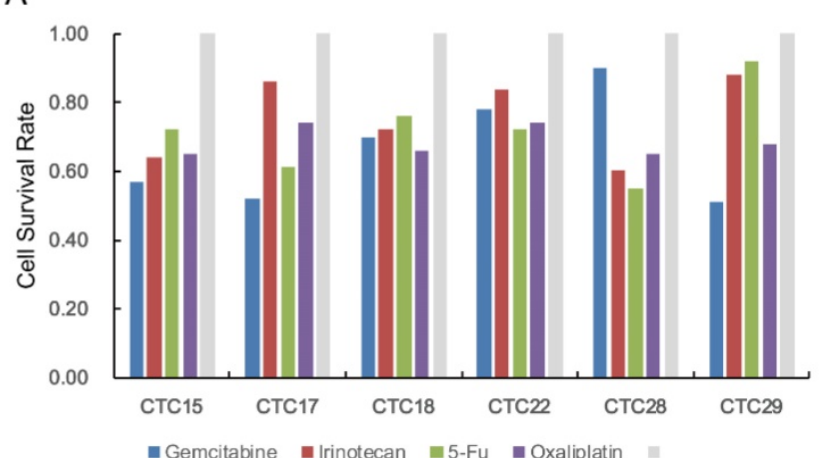

C

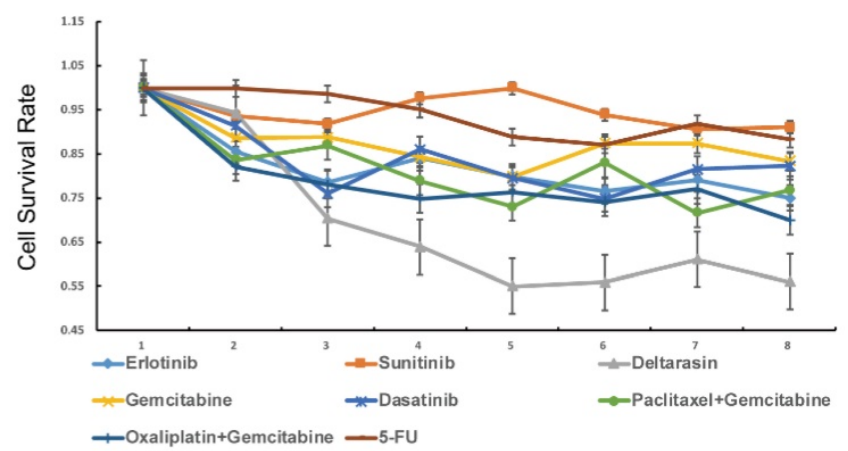

patients with relative high CTCs counts in portal vein (Figure 4A). For cases CTC15, CTC17, CTC18, CTC22, CTC28 and CTC29, we carried out reproducible testing for viability in small numbers of cells (20 50 cells) for identified drug dose. The general results showed that portal vein CTCs was highly drug resistant to several standard clinical regimens (Figure 4A). Here we highlight the drug sensitivity test of case CTC12, a patient with portal vein CTCs over 800 per $7.5 \mathrm{ml}$. Portal vein blood was sampled twice during the infusion chemotherapy (Figure 4B). We tested drug sensitivity of the CTCs using eight kind of clinical regimens or compound. Our results showed that the CTCs was highly resistant to standard clinical regimens such as gemcitabine ,5-FU and gemcitabine plus oxaliplatin (Figure 4C,D). We tested the sensitivity of CTCs to deltarasin, a small molecule inhibited the interaction of RAS with PDE- $\delta$ and suppresses proliferation of human pancreatic cancer cells that are dependent on oncogenic KRAS. The result showed that CTCs viability was reduced after the treatment of deltarasin.

B

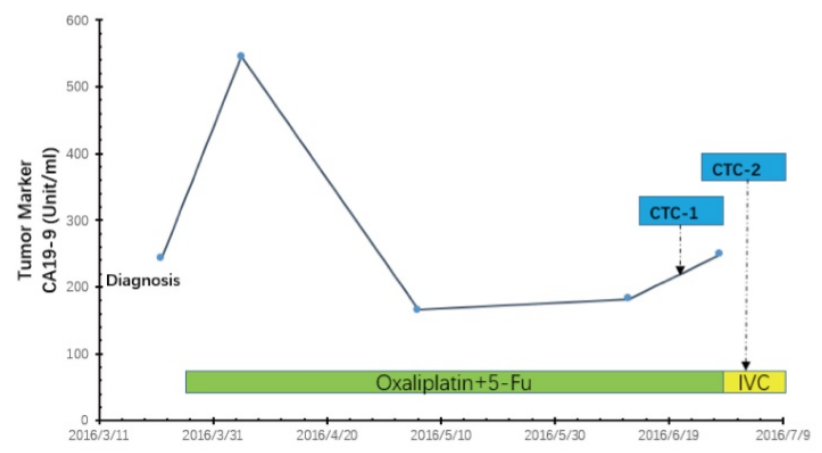

D

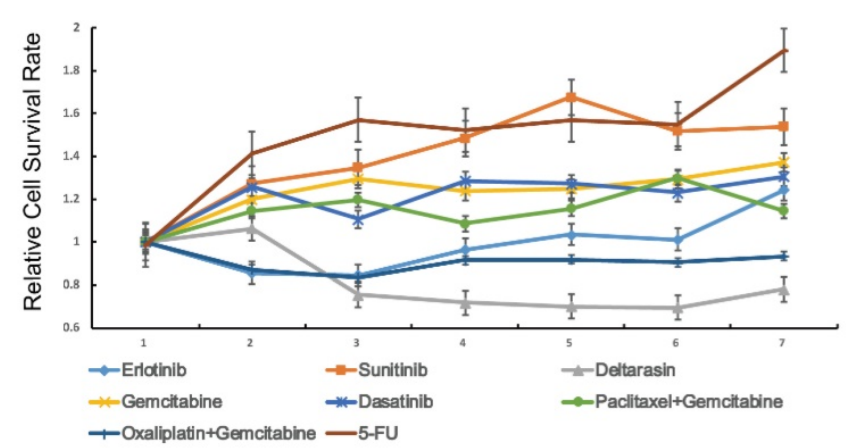

Figure 4. Drug sensitivity test of portal vein CTCs.(A) Drug sensitivity test of case CTC15, CTC17, CTC22,CTC28 and CTC 29 using clinical regimens.(B) Portal Vein CTCs were collected twice during the treatment of case CTC12. (C,D) CTCs derived from case CTC12 was highly resistant to gemcitabine, 5-FU and other clinical regimens, but was sensitive to deltarasin. 


\section{Discussion}

In the present study, we reported that ultrasonography-guided transhepatic puncture was safe and feasible for blood sampling from portal vein in patients with advanced pancreatic cancer. Detection rate and absolute numbers of circulating pancreatic cancer cells was significantly higher in portal vein than that in peripheral circulation. CTCs counts in portal vein was associated with intrahepatic metastases and prognosis of advanced pancreatic cancer. Our results also indicated that circulating pancreatic tumor cells was highly resistant to several standard clinical regimens.

Pancreatic cancer is a kind of systematic disease with complex genetic and epigenetic alternations[31, 32]. Few patients with pancreatic cancer are cured even after radical-intended resection, and up to $80 \%$ patients suffered intrahepatic metastases no more than 2 years[33]. Over the past decades, our knowledge of cancer has increased exponentially, however the understanding of tumor metastases remains poor, especially for pancreatic cancer. In clinic, strategies to treat against intrahepatic metastases of the disease was also extremely limited[6]. Recently, CTCs are found to play critical roles in metastases of various types of cancers[16]. The use of CTCs as a multipurpose tumor biomarker also receives large attraction. Studies of CTCs could increase our understanding of tumor formation, progression, metastasis. At our center, Sun et al reported that CTCs could effectively predict recurrence and prognosis of resectable hepatocellular carcinoma.[22, 34]. Meanwhile, CTCs were also found to be correlated with recurrence, metastases and drug response of breast cancer and prostate cancer[16, 35-37]. A recent meta-analysis reported that CTCs positivity in peripheral circulation was associated with poorer overall survival and progression-free survival in patients with pancreatic cancer [38]. However, it is should be noted that, even for advanced patients, detection circulating pancreatic cancer cells in peripheral circulation remains challenging because of hepatic filtration and technical limitations. In 2015, Catenacci et al first isolated CTCs from portal vein blood [27]. 14 patients with pancreatic cancer were enrolled in their study, all these patients had CTCs detected in portal vein, as opposed to only 3 patients with CTCs detected in peripheral circulation. In view of low detection efficiency for CTCs performed with conventional strategies, portal vein sampling provides a safely and effectively way to detect CTCs in patients with pancreatic cancer. In the present study, we further revealed that CTCs counts in portal vein was highly associated with intrahepatic metastases and prognosis in patients with advanced pancreatic cancer. Overall survival was significant shorter in patients with portal vein CTCs over 150 per $7.5 \mathrm{ml}$ in our cohort. Advanced stage pancreatic cancer comprise a heterogeneous population of patients with remarkable different outcomes. Our results indicated that analysis CTCs in portal vein was an powerful approach for prognosis stratification for patients with advanced pancreatic cancer.

Next, we tried s to test drug sensitivity of CTCs and a panel of single or drug combinations were included. $\mathrm{Yu}$ et al first reported ex vivo culture of circulating breast tumor cells for individualized drug test [17]. Their results indicated that the cultured circulating breast tumor cells was resistant to the standard clinical regimens. Herein, our preliminary results also showed that circulating pancreatic tumor cells were highly resistant to gemcitabine and other common clinical regimens. Drug screening of CTCs derived-from portal circulation enable the identification of highly effective therapeutic drugs for prevention and treatment of intrahepatic metastases. Although the application of portal vein sampling and functional testing of CTCs for individualized treatment of pancreatic cancer is still in its infancy, here we demonstrated its feasibility.

Several major limitations of the study should be noted. First, sample size of the pilot study was relative small. Though portal vein CTCs counts was significant correlated with prognosis of advanced pancreatic cancer in the study, clinical studies with large sample size was still needed to further validate the hypothesis. Second, the relative high cost of portal vein blood collection, CTCs identification and drug test may limit the large-scale clinical study. Third, EpCAM or CK19 positive cells were considered as CTCs in our study, however studies have demonstrated that CTCs, especially the cancer stem cell like CTCs, may loss the epithelial markers due to epithelial-mesenchymal transition. The surface antigen markers of circulating pancreatic cancer cells still need further investigation.

In summary, we demonstrated that in patients with advanced pancreatic cancer, detention of CTCs in portal vein was safe and feasible taking the advantage of ultrasonography-guided transhepatic puncture. CTCs counts in portal vein was significantly higher than that in peripheral circulation, and overall survival was significant shorter in patients with portal vein CTCs over 150 per $7.5 \mathrm{ml}$. CTCs analysis provides a unique tool for studies of metastatic process of pancreatic cancer, as well as mechanisms of drug resistance. 


\section{Abbreviations} aspirates.

CTCs: circulating tumor cells, FNA: fine-needle

\section{Supplementary Material}

Supplementary table S1.

http://www.jcancer.org/v09p2038s1.xlsx

\section{Acknowledgements}

This work was supported by the Rong-Chang Charity Fund of Shanghai Charity Foundation and Zhongshan Hospital Fund for Young Scholars (2015ZSQN31). We are grateful for all staff and subjects participating in the study.

\section{Authors' contributions}

$X Y L$ and YC drafted the manuscript and performed the statistical analyses; WXL, YC, CYL, $\mathrm{LXL}, \mathrm{JHL}, \mathrm{TZY}$ contributed to the sample collection and experiment; GPL, GQG, JMC and WHL contributed to clinical management and following-up of patients; GFZ and YZ assessed the radiographic parameters; WXL, YC and LXL conceived of and designed the study, contributed to manuscript revision and had primary responsibility for final content.

\section{Competing Interests}

The authors have declared that no competing interest exists.

\section{References}

1. Siegel R, Ma J, Zou Z, Jemal A. Cancer statistics, 2014. CA: a cancer journal for clinicians. 2014; 64: 9-29.

2. Seufferlein T, Mayerle J. Pancreatic cancer in 2015: Precision medicine in pancreatic cancer--fact or fiction? Nature reviews Gastroenterology \& hepatology. 2016; 13: 74-5.

3. Yachida S, Jones S, Bozic I, Antal T, Leary R, Fu B, et al. Distant metastasis occurs late during the genetic evolution of pancreatic cancer. Nature. 2010; 467: 1114-7.

4. Yang KS, Im H, Hong S, Pergolini I, Del Castillo AF, Wang R, et al. Multiparametric plasma EV profiling facilitates diagnosis of pancreatic malignancy. Science translational medicine. 2017; 9 .

5. Garrido-Laguna I, Hidalgo M. Pancreatic cancer: from state-of-the-art treatments to promising novel therapies. Nature reviews Clinical oncology. 2015; $12: 319-34$

6. Von Hoff DD, Ervin T, Arena FP, Chiorean EG, Infante J, Moore M, et al. Increased survival in pancreatic cancer with nab-paclitaxel plus gemcitabine. The New England journal of medicine. 2013; 369: 1691-703.

7. Nagakawa Y, Hosokawa Y, Nakayama H, Sahara Y, Takishita C, Nakajima T, et al. A phase II trial of neoadjuvant chemoradiotherapy with intensity-modulated radiotherapy combined with gemcitabine and S-1 for borderline-resectable pancreatic cancer with arterial involvement. Cancer chemotherapy and pharmacology. 2017; 79: 951-7.

8. Deplanque G, Demartines N. Pancreatic cancer: are more chemotherapy and surgery needed? Lancet (London, England). 2017; 389: 985-6.

9. Uesaka K, Boku N, Fukutomi A, Okamura Y, Konishi M, Matsumoto I, et al. Adjuvant chemotherapy of S-1 versus gemcitabine for resected pancreatic cancer: a phase 3, open-label, randomised, non-inferiority trial (JASPAC 01). Lancet (London, England). 2016; 388: 248-57.

10. Jordan NV, Bardia A, Wittner BS, Benes C, Ligorio M, Zheng Y, et al. HER2 expression identifies dynamic functional states within circulating breast cancer cells. Nature. 2016; 537: 102-6.

11. Massague J, Obenauf AC. Metastatic colonization by circulating tumour cells. Nature. 2016; 529: 298-306.

12. Shen $\mathrm{Z}, \mathrm{Wu} \mathrm{A}, \mathrm{Chen} \mathrm{X}$. Current detection technologies for circulating tumor cells. Chemical Society reviews. 2017; 46: 2038-56.
13. Gao D, Vela I, Sboner A, Iaquinta PJ, Karthaus WR, Gopalan A, et al. Organoid cultures derived from patients with advanced prostate cancer. Cell. 2014; 159: 176-87.

14. Kolostova K, Rzechonek A, Schutzner J, Grill R, Lischke R, Hladik P, et al. Circulating Tumor Cells as an Auxiliary Diagnostic Tool in Surgery. In vivo (Athens, Greece). 2017; 31: 1197-202.

15. Micalizzi DS, Maheswaran S, Haber DA. A conduit to metastasis: circulating tumor cell biology. Genes \& development. 2017; 31: 1827-40.

16. Aceto N, Bardia A, Miyamoto DT, Donaldson MC, Wittner BS, Spencer JA, et al. Circulating tumor cell clusters are oligoclonal precursors of breast cancer metastasis. Cell. 2014; 158: 1110-22.

17. Yu M, Bardia A, Aceto N, Bersani F, Madden MW, Donaldson MC, et al. Cancer therapy. Ex vivo culture of circulating breast tumor cells for individualized testing of drug susceptibility. Science (New York, NY). 2014; 345: 216-20.

18. Vogelzang NJ, Fizazi K, Burke JM, De Wit R, Bellmunt J, Hutson TE, et al. Circulating Tumor Cells in a Phase 3 Study of Docetaxel and Prednisone with or without Lenalidomide in Metastatic Castration-resistant Prostate Cancer. European urology. 2017; 71: 168-71.

19. Goldkorn A, Ely B, Tangen CM, Tai YC, Xu T, Li H, et al. Circulating tumor cell telomerase activity as a prognostic marker for overall survival in SWOG 0421: a phase III metastatic castration resistant prostate cancer trial. International journal of cancer. 2015; 136: 1856-62.

20. Goldkorn A, Ely B, Quinn DI, Tangen CM, Fink LM, Xu T, et al. Circulating tumor cell counts are prognostic of overall survival in SWOG S0421: a phase III trial of docetaxel with or without atrasentan for metastatic castration-resistant prostate cancer. Journal of clinical oncology: official journal of the American Society of Clinical Oncology. 2014; 32: 1136-42.

21. Goodman OB, Jr., Symanowski JT, Loudyi A, Fink LM, Ward DC, Vogelzang NJ. Circulating tumor cells as a predictive biomarker in patients with hormone-sensitive prostate cancer. Clinical genitourinary cancer. 2011; 9: 31-8.

22. Sun YF, Xu Y, Yang XR, Guo W, Zhang X, Qiu SJ, et al. Circulating stem cell-like epithelial cell adhesion molecule-positive tumor cells indicate poor prognosis of hepatocellular carcinoma after curative resection. Hepatology. 2013; 57: 1458-68.

23. Ogle LF, Orr JG, Willoughby CE, Hutton C, McPherson S, Plummer R, et al. Imagestream detection and characterisation of circulating tumour cells - A liquid biopsy for hepatocellular carcinoma. Journal of hepatology. 2016; 65: 305-13.

24. Vakoc CR, Tuveson DA. Untangling the genetics from the epigenetics in pancreatic cancer metastasis. Nature genetics. 2017; 49: 323-4.

25. Maddipati R, Stanger BZ. Pancreatic Cancer Metastases Harbor Evidence of Polyclonality. Cancer discovery. 2015; 5: 1086-97.

26. Nagrath S, Jack RM, Sahai V, Simeone DM. Opportunities and Challenges for Pancreatic Circulating Tumor Cells. Gastroenterology. 2016; 151: 412-26.

27. Catenacci DV, Chapman CG, Xu P, Koons A, Konda VJ, Siddiqui UD, et al. Acquisition of Portal Venous Circulating Tumor Cells From Patients With Pancreaticobiliary Cancers by Endoscopic Ultrasound. Gastroenterology. 2015; 149(e4): 1794-803

28. Poruk KE, Blackford AL, Weiss MJ, Cameron JL, He J, Goggins M, et al. Circulating Tumor Cells Expressing Markers of Tumor-Initiating Cells Predict Poor Survival and Cancer Recurrence in Patients with Pancreatic Ductal Adenocarcinoma. Clinical cancer research: an official journal of the American Association for Cancer Research. 2017; 23: 2681-90.

29. Chudasama DY, Freydina DV, Freidin MB, Leung M, Montero Fernandez A, Rice A, et al. Inertia based microfluidic capture and characterisation of circulating tumour cells for the diagnosis of lung cancer. Annals of translational medicine. 2016; 4: 480.

30. Garnett MJ, Edelman EJ, Heidorn SJ, Greenman CD, Dastur A, Lau KW, et al. Systematic identification of genomic markers of drug sensitivity in cancer cells. Nature. 2012; 483: 570-5.

31. Jakel C, Bergmann F, Toth R, Assenov $Y$, van der Duin D, Strobel O, et al Genome-wide genetic and epigenetic analyses of pancreatic acinar cell carcinomas reveal aberrations in genome stability. Nature communications. 2017; 8: 1323.

32. Cancer Genome Atlas Research Network. Electronic address aadhe, Cancer Genome Atlas Research N. Integrated Genomic Characterization of Pancreatic Ductal Adenocarcinoma. Cancer cell. 2017; 32(e13): 185-203.

33. $\mathrm{Yu} \mathrm{X}, \mathrm{Gu} \mathrm{J}, \mathrm{Fu} \mathrm{D}$, Jin C. Dose surgical resection of hepatic metastases bring benefits to pancreatic ductal adenocarcinoma? A systematic review and meta-analysis. International journal of surgery. 2017; 48: 149-54.

34. Guo W, Yang XR, Sun YF, Shen MN, Ma XL, Wu J, et al. Clinical sionificance of EpCAM mRNA-positive circulating tumor cells in hepatocellular carcinoma by an optimized negative enrichment and qRT-PCR-based platform. Clinical cancer research: an official journal of the American Association for Cancer Research 2014; 20: 4794-805.

35. Baccelli I, Schneeweiss A, Riethdorf S, Stenzinger A, Schillert A, Vogel V, et al. Identification of a population of blood circulating tumor cells from breast cancer patients that initiates metastasis in a xenograft assay. Nature biotechnology. 2013; 31: 539-44.

36. Boral D, Vishnoi M, Liu HN, Yin W, Sprouse ML, Scamardo A, et al. Molecular characterization of breast cancer CTCs associated with brain metastasis. Nature communications. 2017; 8: 196. 
37. Sonpavde G, Antonarakis ES. Circulating Tumor Cells in Advanced Prostate Cancer: Time to Move from Prognostic to Predictive Ability. European urology. 2017; 71: 172-3.

38. Han L, Chen W, Zhao Q. Prognostic value of circulating tumor cells in patients with pancreatic cancer: a meta-analysis. Tumour biology: the journal of the International Society for Oncodevelopmental Biology and Medicine. 2014; 35: 2473-80. 